Clinical guidelines

\title{
The management of anticoagulation in patients with prosthetic heart valves undergoing non-cardiac operations
}

\author{
Walter J Busuttil, Brian M Fabri
}

\begin{abstract}
Summary
Prosthetic valve thrombogenicity and bleeding complications associated with life-long anticoagulation are constant potential causes of morbidity and mortality following prosthetic valve implantation. The conflict between over- and under-anticoagulation is even more of a problem when other surgical interventions are required. Very few clinical trials have addressed this issue. We propose some guidelines based on the concept of risk-adjusted intensity of anticoagulation but stress the need for caution with intepretation of these recommendations.
\end{abstract}

Keywords: anticoagulation, prosthetic valves, non-cardiac surgery

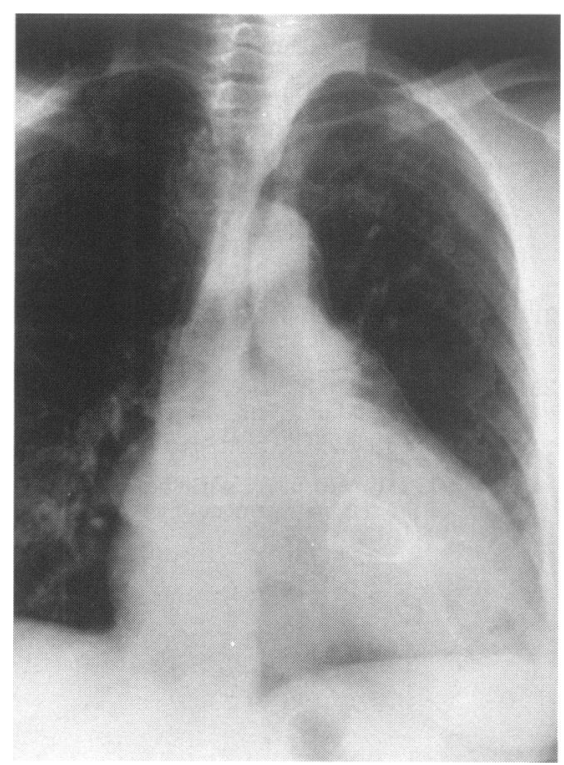

Figure 1 Starr-Edwards mitral valve prosthesis

The Cardiothoracic Centre, Thomas Drive, Liverpool L14 3PE, UK

WJ Busuttil

BM Fabri

Correspondence to BM Fabri

Accepted 2 February 1995
Thrombosis, embolism, and bleeding remain the most important late complications after heart valve replacement. ${ }^{1}$ If such patients need to undergo other surgical interventions the management of their anticoagulation is complicated by the conflict between over-and under-anticoagulation in relation to the prosthetic valve(s) and the proposed intervention. Unfortunately there is a paucity of data addressing this issue $\mathrm{e}^{2,3}$ and therefore most of the views regarding the best form of management are not based on clinical trials but rather on traditional and arbitrary practices. ${ }^{4}$

There are several reasons why trials have not been carried out more extensively. Firstly, it is fair to say that, in general, clinicians often hold pre-set views as to the best form of management of a clinical problem even if there is no scientific proof of this being the optimal method. This certainly applies to the management of anticoagulation where varying attitudes are often based on previous clinical experiences not backed by accurate scientific data. Secondly, the risk factors for thromboembolic complications are numerous and varied and therefore a homogenous study population is very difficult to set up. Finally, the number of thromboembolic and/or bleeding events is usually small and therefore large scale multicentre studies would be required to address this issue.

Because of this lack of scientific data these guidelines can only be put forward in cautious terms. They are based on a review of the literature. However, it must be stressed that they can only be considered as recommendations because they are heavily biased by clinicians' views and experiences.

\section{Risk factors for thrombosis and embolism}

Multiple factors contribute to the pathogenesis of thrombosis and embolism. They can be interpreted through the classical hypothesis of Virchow's triad (box 1) and can also be broadly classified into risk factors associated with the patient (box 2$)^{5,6}$ and risk factors associated with the valve prosthesis (box 3 ). In addition, complications of valve thrombosis and systemic embolisation following the implantation of artificial heart valves are related to the type and design as well as location of the prosthesis.

\section{Valve prostheses}

Valve prostheses can be broadly classified into mechanical and bioprosthetic valves (box 4). Mechanical valves are more thrombogenic than biological valves. The newer second generation valves are in turn less thrombogenic than the older mechanical valves. The site of implantation of the prosthesis is also important in determining thrombogenicity. Thus, replacement valves in the mitral position are associated with a higher incidence of embolism than those in the aortic position. ${ }^{7}$

The European Society of Cardiology has recently published guidelines for the prevention of thromboembolic events in valvular heart disease. ${ }^{4}$ Long-term anticoagulation is recommended in all patients with mechanical valve replacements and in all patients with valvular heart disease who are in chronic or paroxysmal atrial fibrillation. In patients with an aortic bioprosthesis who are in sinus rhythm, once the sewing ring is endothelialised at around three months and in the absence of a previous history of emboli or rhythm disturbances, long term anticoagulation is unnecessary. In the mitral position, however, long-term anticoagulation should be considered in cases with a persistently large left atrium $(>50 \mathrm{~mm})$, persistent heart failure or marked cardiomegaly. Long-term 


\begin{tabular}{|l|}
\hline Virchow's triad \\
\hline - changes in the vessel wall (or \\
surface of the valve) \\
changes in the local pattern of blood \\
flow \\
- changes in the constituents of blood \\
\hline
\end{tabular}

Box 1

\begin{tabular}{|l|}
\hline Risk factors associated with \\
the patient \\
\hline age \\
- atrial fibrillation \\
enlarged left atrium \\
- heart failure \\
- increased coagulability of the blood \\
hyperlipidaemia \\
cigarette smoking \\
hypertension \\
diabetes \\
drugs, eg, oral contraceptives \\
malignant disease \\
nephrotic syndrome \\
\hline
\end{tabular}

Box 2

\section{Risk factors associated with the prosthesis}

- type: mechanical valves

- design: first generation mechanical valves

- location: mitral position

Box 3

\begin{tabular}{|l|}
\hline Prosthetic heart valves \\
\hline Biological valves \\
- pericardial \\
- porcine \\
Mechanical valves \\
- 1st generation \\
ball cage: Starr-Edwards \\
tilting disc: Bjork Shiley \\
$\quad$ Omniscience \\
- 2nd generation \\
tilting disc: Medtronic \\
$\quad$ Monostrut \\
bileaflet: St Jude Medical \\
Carbomedics \\
\hline
\end{tabular}

Box 4

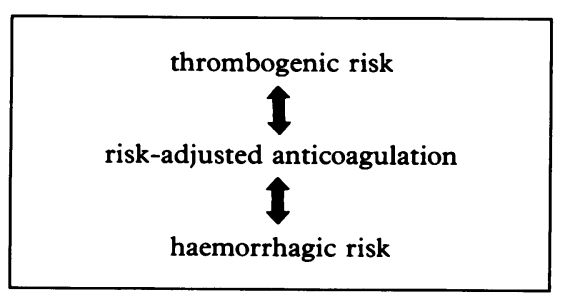

Box 5 anticoagulation is also necessary in those with a history of systemic emboli, left atrial thrombus at surgery, and in those in whom atrial fibrillation re-occurs.

\section{Intensity of anticoagulation}

Because of the large number of factors affecting thrombogenicity Butchart et al ${ }^{8}$ have proposed the concept of risk-adjusted intensity of anticoagulation (box 5). Since the likelihood of bleeding complications increases with a higher target range of anticoagulation, attempts have been made to identify the optimum level of anticoagulation. Recent studies ${ }^{9-13}$ have shown that, with certain valve types, reducing the intensity of anticoagulation has reduced haemorrhagic complications without an increase in the incidence of thromboembolism.

The European Society of Cardiology ${ }^{4}$ has recently recommended target ranges of anticoagulation for various prostheses (box 6). Adjustments to these recommendations may be required in relation to patient-related embolic risk factors.

\section{Guidelines}

Although thrombosis and embolism can occur even during optimal levels of anticoagulation, there is no doubt that reducing the level of anticoagulation below an acceptable level will lead to an increase in thrombotic and embolic problems. ${ }^{13}$ Valve thrombosis is a potentially fatal complication and great care must be taken before recommending that anticoagulation be reduced. We therefore propose that Butchart's concept of risk-adjusted anticoagulation be extended to the management of anticoagulation during non-cardiac surgery (figure 2). Risk-adjusted management needs to take two factors into consideration:

1 The patient and valve thrombogenicity, ie, the thrombogenic risk. The importance of valve type, valve site, previous history of embolism, presence of atrial fibrillation, large left atrium, etc, have already been discussed. Obviously the degree of reduction of anticoagulation for surgery should be related to the risk of thrombotic and embolic phenomena.

2 The operation and the risks of bleeding, ie, the haemorrhagic risk. Careful haemostasis by experienced surgeons will allow most operations to be carried out safely with an acceptable level of anticoagulation.

In patients in whom the thrombotic risk is extremely high we suggest that anticoagulation is not stopped. Oral anticoagulation should be converted to intravenous anticoagulation with a heparin infusion. There are several advantages to this. Heparin has a much shorter half-life than warfarin and therefore fine adjustment of anticoagulation levels is easier. Reversal of heparin using protamine in case of a surgical catastrophe is also much easier. In the case of patients who have a very high haemorrhagic risk the intravenous administration of heparin can be stopped three hours before the start of surgery and then restarted as soon as haemostasis is secured.

In patients with a low haemorrhagic risk we would suggest that as long as the levels of anticoagulation are within the guidelines recommended, surgery can be carried out safely without exposing the patient to thrombotic complications. We propose that, in these patients, oral anticoagulation should be continued during the pre- and post-operative period.

In patients who have a low thrombogenic valve implanted and in whom thrombogenic risks are low, it has been shown by some studies ${ }^{3,14}$ that it is possible to stop anticoagulation completely for several days pre- and postoperatively without incurring excessive risk of thrombosis and embolism. Unfortunately, these studies have only analysed comparatively small numbers of patients. It is our opinion that the serious risks associated with stopping anticoagulation far outweigh the reduced risks of bleeding complications and doing so would be exposing the patient to a totally unnecessary risk of death.

In patients with a high thrombogenic risk the following plan of action is proposed. The patient should be admitted two days pre-operatively, having been asked to stop warfarin three days pre-operatively. This is based on the half-life of warfarin which varies from $18-36$ hours. ${ }^{15}$ Heparinisation is started using a continuous infusion aiming to maintain an anticoagulation level in the therapeutic range (an activated partial thromboplastin time (APPT) of 60-80 s). Frequent APPT estimates (six hourly) are necessary to achieve satisfactory levels of anticoagulation. In patients with a higher haemorrhagic risk the heparin should be stopped three hours before the start of the operation while in patients 


\section{Target ranges for anticoagulation}

- first generation mechanical valves (Starr-Edwards, Björk-Shiley Standard, Omniscience):

INR: $3.0-4.5$

- second generation bileaflet valves (St Jude, Carbomedics) and tilting disc valves (Medtronic,

Monostrut)

mitral position INR: 3.0

aortic position INR: 2.5

Box 6

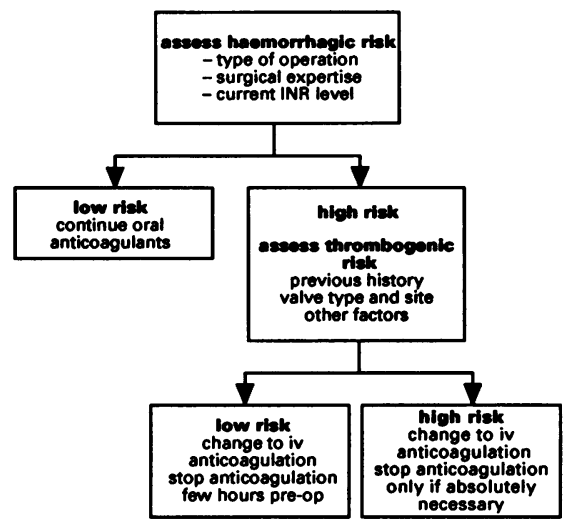

Figure 2 in whom the haemorrhagic risk is assessed to be low anticoagulation should not be stopped at all. Heparinisation is restarted per-operatively as soon as the risks of major haemorrhage have been overcome and only stopped once the levels of oral anticoagulation have returned into the recommended therapeutic range.

We emphasise that these recommendations are indeed just guidelines that can and should be modified depending on the balance between thrombogenic and haemorrhage risks.

We would further recommend that:

- patients with prosthetic heart values are in the majority of cases unsuitable for day case surgery

- the surgery should be carried out by experienced surgeons since the need for reducing anticoagulation and the haemorrhagic risks are obviously related to technical ability

- limited access procedures in patients who are anticoagulated are not to be recommended

- the availability of blood product volume replacement for these patients should be ensured

- in cases of emergency operations fresh frozen plasma is effective in neutralising oral anticoagulation but we suggest that intravenous anticoagulation should be started as soon as it is safe to do so.

Unfortunately these recommendations are not based on scientific clinical trials but rather on individual convictions and beliefs. These tentative recommendations and cautious terminology stress the need for trials in order to outline the optimal method(s) of management.

1 Acar J. Thromboembolic events in prosthetic valve recipients: What is the safe level of anticoagulation? (editorial) $f$ Heart Valve Dis 1993; 2: 395-7.

2 Katholi RE, Nolan SP, McGuire LB. The management of anticoagulation during non cardiac operations in patients with prosthetic hear values: a prospective study. Am Heart $\mathcal{f} 1978$ 96: $163-5$.

3 Darman D, Enriquez-Sarano M, Acar J. Cardiac complications after subsequent non cardiac operations in patients with non-biological prosthetic heart values. Eur Heart $f$ 1983; 4 (suppl 1).

4 Ad Hoc Committee of the Working Group on

Valvular Heart Disease, European Group on Valvular Heart Disease, European Society of boembolic events in valvular heart disease. $f$ Heart Valve Dis 1993; 2: 398-410.

5 Abernathy WAS, Willis PW. Thromboembolic complications of rheumatic heart disease. Valcomplications of rheumatic hear
vular Heart Dis 1973; 5: 132 .

6 vular Heart Dis 1973; 5: 132. $\mathrm{RW}$. Systemic embolisation in mitral stenosis. Br Heart F 1964; 26: 1393.

7 Edmunds LH Jr. Thrombotic and bleeding complications of prosthetic heart valves. $A n n$ Thorac Surg 1987; 44: 430-45.

8 Butchart EG, Lewis PA, Bethal JA, Breckenridge IM. Adjusting anticoagulation to prosthesis thrombogenicity and patient risk factors. Circulation 1991; 84 (suppl III): III-61 - III-69.

9 Turpie AGG, Gunstensen J, Hirsh J, Nelson H,

Gent $M$. Ranisierter vergleich Gent $M$. Randomisierter vergleich zweie therapie nach bioklappenersatz. Lancet 1988; 2, 587-90.
10 Altman R, Rouvier J, Gurfinkel E, et al. Comparison of two levels of anticoagulation therapy in patients with substitute heart valves. $\mathcal{F}$ Thorac Cardiovasc Surg 1976; 72: 127-9.

11 Saour JN, Sieck JO, Mamo LAR, Gallus AS. Trial of different intensities of anticoagulation in patients with prosthetic heart valves. $N$ Engl $\mathcal{F}$ Med 1990; 332: 428-32.

12 Horstkotte D, Schulte HD, Bircks W, Strauer $B E$. Unexpected findings concerning thromboembolic complications and anticoagulation after complete ten year follow up of patients with $S$ Jude Medical prostheses. $₹$ Heart Valve Dis 1993; 2: 291-301.

13 Butchart EG, Lewis PA, Grukemeier GL Butchart EG, Lewis PA, Grukemeier GL Kulatilake N, Breckenridge I. Low risk of thrombosis and serious embolic events despite airculation 1988; 78: suppl I: $67-76$

14 Acar J, Enriquez-Sarano M, Farah E, Kassab R, Tubian P, Roger V. Recurrent systemic embolic events with valve prosthesis. Eur Heart $\mathcal{F} 1984 ; 5$ (suppl D): 33-8.

14 Tinker TH, Taraham S. Discontinuing anticoagulant therapy in surgical patients with cardiac valve prostheses: observations in 180 patients. F $A M A$ 1978; 239; 738-9.

15 Shetty HGM, Woods F, Routledge PA. The pharmacology of oral anticoagulants: implications for therapy. $\mathcal{F}$ Heart Valve Dis 1993; 2: $53-62$. low intensity of anticoagulation. Experience 